\title{
Passive Adaptive Network Fuzzy Inference Controller Based wheeled Mobile Robot
}

\author{
K. SUGANYA \\ Department of Research- Ph.D Computer Science, Tiruppur \\ Kumaran College for Women, Tiruppur, INDIA
}

\author{
V. ARULMOZHI \\ Department of Research-Ph.D Computer Science, Tiruppur \\ Kumaran College for Women, Tiruppur, INDIA
}

\begin{abstract}
Passive adaptive network fuzzy inference controllerby teleoperation based wheeled mobile robot navigationand wheel slippage has been envisaged in this paper. Apassivity controller mode is employed to render the obstacleavoidance for wheeled mobile robot thereby ensuring incidentfree navigation particularly unlimited workspace andsurface slippage of coordination of master robot positionand slave robot velocity. In order to achieve system stabilityeffective control strategies has been formulated. Thesestrategies bypass robot slippage and skidding due to softcomputing.Perfect position tracking of the master/ slaverobot with Simpson 1/3 rule based adaptive neural networkfuzzy inference system is reducing the error in terms ofunavoidable and acceptable forces, which is confirmed bypassivity. We demonstrated theoretically, the systems stabilityis shown via its passivity hybrid system in that force feltby the human operator is approximately equal to the forcesapplied by the environment force and sensor predictor ofslave robot, which is a satisfactory performance outcome.Simulation results verify the effectiveness of the proposedscheme for teleoperation under passivity controller.
\end{abstract}

Key-Words: Autonomous wheeled mobile robot, Passivity, adaptive network fuzzy inference system,Simpson's 1/3 rule, teleoperation system.

Received: May 3, 2020. Revised: September 5, 2020, Accepted: October 15, 2020. Published: November 17, 2020.

\section{Introduction}

Wheeled mobile robot (WMR) is travelling on a wheel slippage surfaces (or) soft surface, due to obstacles is a challenging task the perfect and pure rolling of wheels. This wheel slippage and obstacles affect the WMR's dynamic models, creating challenges for control. Teleoperation based WMR is more useful when robot tracking can enhance the navigation and control capability, in this paper we consider the problem of WMRs unlimited workspace and surface slippage of coordination of master robot position $q_{m}$ and slave robot velocity $\dot{q}_{s}$, ideal assumption of pure rolling (zero slippage) in case of twowheeled mobile robot that travels forward/backward with free motion at any rotation/directions. We present that owing to the WMR compensate for designing the control system, the visual feedback from the lacking slave robot prediction, is added to teleoperation architecture. It is response to the operators commands using while the operator can regulate his/him commands effectively. In particular, the master robot interacts with human operator and slave robot is predicting wheel slippery/pure rolling as based on Simp- sons $1 / 3$ rule base adaptive network fuzzy inference system (SANFIS) and interacting with its own environment.

In wheeled mobile robot teleoperation there are two kinematics-related challenges not often experienced, which is (i) the workspace of the master robot is limited, visa verse the slave robot is often much bigger and (ii) the WMR with non-holonomic constraints are considered to move in any direction but they are not introduced any variable for direction in the scope of interlink wheel slippage and skidsteering effects [1][2]. The coordination between master and slave robot position and velocity employing bilateral tele-drive of WMRs with unlimited workspace. However, WMR on loose soil, disaster exploration and scientific expedition always require workspace mismatch and surface slippage at the time of actuated mobile robot position and desired angular velocity. These kinds of subjects are still open and remain challenges to achieve workspace mismatch and surface slippage, i.e. collision-free path while moving 
towards goal.

The time delay power network method was introduced in [5] to present the mobile robot with teleoperation tracking navigations. This system is force feedback of delayed network with passivity and also obstacle based force feedback which directly relates the distance between the mobile robot and obstacles. It may be noted that the force calculation is not often in slave mode controls, which are interacting with environment forces. The time domain passivity network approach is introduced in [6], in order to restore zero velocity of the mobile robot. This type of force feedback has two important roles: (i) A human-operator facilitating sensor data communication thereby increasing velocity of slave robot; (ii) Restoring data position of the master device during stopping causes longitudinal slippage. If the slave velocity is too fast and if direction changes occur frequently, robots may fall down. Here velocity controller of slave robot cannot proceed due to existing external disturbances.

In [11]-[17] rich theory developed owing to WMRs achieve a passivity within unlimited workspace, Cartesian coordinates with non-holonomic constraints and semi-autonomous control strategy. However, WMRs kinematic model with embedded controller ensuring tracking desired angular velocity invertible errors between desired and actual linear angular velocity. The case of wheel slippage, the WMRs velocity level controller assumed as $s_{1}=\frac{\gamma \omega_{1}}{v_{1}}, s_{2}=\frac{\gamma \omega_{2}}{v_{2}}$, where $s_{1}$ is the left wheel slippage, $s_{2}$ is right wheel slippage, $r$ is radius of the wheels, wheels actual linear velocity $v_{1}(l e f t), v_{2}($ right) and wheel desired angular velocity $\omega_{1}(l e f t)$, and $\omega_{2}$ (right). In this paper, synchronized joint position error: master robot desired angular velocity minis slave robot actual angular velocity does not reconnected to the real system. And also does not given any mathematical proof as well as stability proof, because most of time predictor error will give more convenient perfect tracking. In this respect the reference [13]-[15] is lacking. We should fulfilling the gap, we considered the concept of passivity controller as per Simpson's $1 / 3$ rule based adaptive network fuzzy influence with teleoperation system. This method shows wheel moving energy, velocity and angular velocity reallocate direct to target point, then the robot moves in forward on straight line of target from obstacles and investigate environment effect.

The presented article is prepared as follows: Section II gives the preliminary formulation of problem. In Section III, the design of control section IV gives stability analysis of dynamical system. The proposed controllers are implemented in simulation results for path planning in Section V. The conclusion is given in Section VI.

Notation: In this paper, $\mathcal{R}$ denotes the real numbers and $\mathcal{C}$ denotes the complex numbers, $C^{1}$ is continuous differentiable on some closed time interval. For any $X-Y$ direction the robot can move freely with different environment effects. The desired angle with respect of initial linear velocity $V$ and initial angular velocity $U$ at target point with respect to vehicles absolute positions and measure.

\section{Problem Formulation and Preliminaries}

In this paper, we consider two-wheeled mobile robot type, the back two wheels are driving wheels and front wheel is free. Thus, WMR can rotate at any direction with respect of up and down of steering wheels. Then bloc diagram of path following strategy is showing in Fig.1.

\subsection{Dynamic model of mobile robot}

In order to describe position of wheeled mobile robots, two different coordinates are needed.

The master robot (or) reference frame of wheeled 
mobile robot dynamical system is

$$
\begin{aligned}
M_{m}\left(q_{m}\right) \ddot{q}_{m} & +C_{m}\left(q_{m}, \dot{q}_{m}\right) q_{m}+g_{m}\left(q_{m}\right)+\tau_{d m} \\
& =\tau_{m}+\tau_{h},
\end{aligned}
$$

where $q_{m}, \dot{q}_{m}, \ddot{q}_{m}$ are master robot (or) reference frame position, velocity and acceleration respectively. $M_{m} \in$ $R^{m} \times R^{m}$ be inertial matrices of reference frame, $C_{m}\left(q_{m}, \dot{q}_{m}\right) \in R^{m} \times R^{m}$ be coriolis and centrifugal effects, $\tau_{d m} \in R^{l_{m}}$ is desired future predicted input of master frame(i.e. error response of slave robot + master robot new control command), $g_{m}\left(q_{m}\right) \in R^{m}$ is gravitational forces, $\tau_{h} \in R^{m}$ is fixed environment effect, $\tau_{m} \in R^{m}$ is control signal reference frame.

The slave robot (or) local frame of wheeled mobile robot dynamical system is

$$
\begin{aligned}
M_{s}\left(q_{s}\right) \ddot{q}_{s} & +C_{s}\left(q_{s}, \dot{q}_{s}\right) q_{s}+g_{s}\left(q_{s}\right)+\tau_{d s} \\
& =\tau_{s}-A^{T}\left(q_{s}\right) \lambda-\tau_{e},
\end{aligned}
$$

where $q_{s}, \dot{q}_{s}, \ddot{q}_{s}$ are robot wheel skid-steering position, wheel velocity and acceleration respectively. $M_{s} \in R^{n} \times R^{n}$ be entire wheel inertial matrices after skidding, $C_{s}\left(q_{s}, \dot{q}_{s}\right) \in R^{n} \times R^{n}$ be coriolis and centrifugal effects with skidded-steering condition, $g_{s}\left(q_{s}\right) \in R^{n}$ is gravitational at skidded-steering condition, $\tau_{s d} \in R^{l_{n}}$ is desired future predicted input of slave robot (i.e. error response of master robot + camera capture slave robot new control command), $\lambda$ is unknown parameter, $\tau_{e} \in R^{n}$ is environmental forces and $\tau_{s} \in R^{n}$ is control signal at skid-steering vehicle and thus moving with respect of master control reference frame within it.

\subsection{WMR ssynchronized model with actual robot linear velocity and desired angle}

The Fig. 1 shows forward direction of WMR x-axis position where $\Theta_{c}$ is equal to camera capture of desired angular velocity minis predicted angular velocity, $\Theta_{d}$ is equal to desired linear velocity minis actual linear velocity. While $A$ is center point of wheel mobile robot and commented angle of right/left wheel is

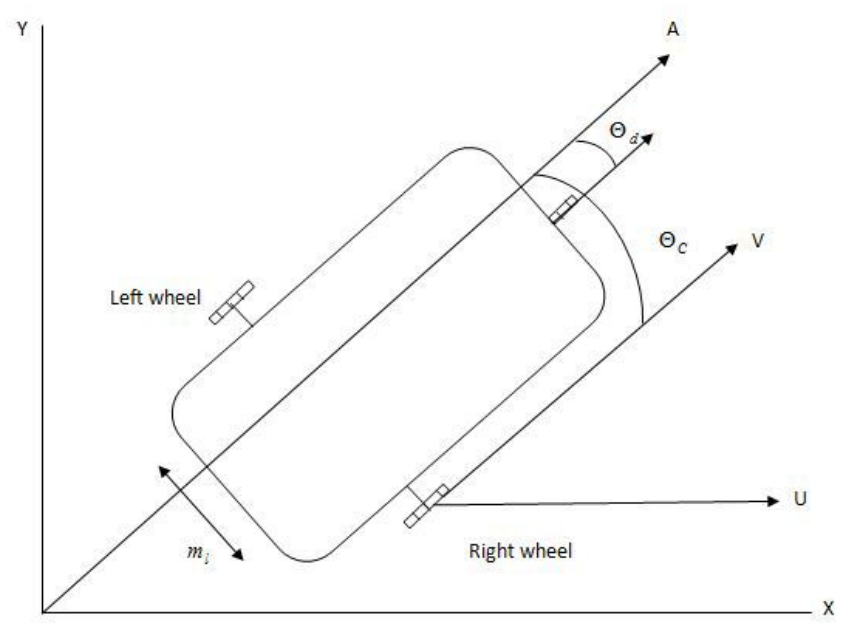

Fig. 1. Path following strategy.

$\theta=\Theta_{d}-\Theta_{c}$ which is interlinking predictor velocity of slave robot with respect of wheel radius $r$ and width $m_{l}$.

Remark 1 If $\Theta_{c}$ is equal to $\Theta_{d}$, then $\theta$ is equal to zero and therefore wheels get pure rolling with respect of wheel radius $r$. The difficulty is obtaining the exact position and wheel slippage, in [15]the position is not directly used in computer error feedback loop controller, so it is expected that the trajectory tracking performance will not affected too much.

However, we coupled the WMR slippage with environment affect and slave robot response. Define sensor detected at right/left wheel is $\dot{\theta}=\dot{\Theta}_{i}+\tau_{i}$, $i=m, s$, where $\Theta_{i}$ is defined later and actual wheel slippage is not decided by states of WMR, it also depending external environment. On behalf off we consider control input $\tau_{i}=\hat{\tau}_{i}-\bar{\tau}_{i}, i=m, s$ where $\bar{\tau}_{i}, i=m, s$ predicted state input vectors of master/slave robot, $\hat{\tau}_{i}$ is regulated out, it means that out error coming from WMR response. Define the future prediction control input $\tau_{d_{i}}=f\left(\dot{\Theta}_{i}, \dot{\theta}, \tau_{i}\right)$ and error between future prediction position and WMR actual position is denoted as $e_{i} \in R^{o}$. In this case we consider future prediction position will be used in Hybrid system $\mathcal{H}_{\tau_{d_{i}}}$, then $\mathcal{H}_{\tau_{d_{i}}}$ can be written in the 
suggestive from:

$$
\begin{aligned}
& \tau_{d_{i}}=f\left(\theta, \Theta_{i}, \tau_{i}\right), \forall \\
& \left(\tau_{d_{i}}, \theta, \Theta_{i}, \tau_{i}\right) \in R^{l_{j}} \times R^{m+1 \times n+1} \\
& \times R^{m \times n} \times R^{i}, j=m, n, i=m, s .
\end{aligned}
$$

with output

$$
\theta=\Theta_{i}+\tau_{i}
$$

$f: C_{m \times n} \times D_{m \times n} \rightarrow R$ is semi continuous and locally bounded on the compact intervals of $\mathcal{B}$, the inputs $\tau_{m}, \tau_{s}$ and $\tau_{d_{i}}, i=m, s$ exists in $f$ and without restrictions on the initial state and non zero restriction on the input and with linear asymptotic gain satisfies the Lipschitz condition, then stabilizer of the WMR is taken linear as

$$
f^{\prime}\left(\theta, \Theta_{i}, \tau_{i}\right)=\frac{\partial f\left(\theta, \Theta_{i}, \tau_{i}\right)}{\partial \theta}+\frac{\partial f\left(\theta, \Theta_{i}, \tau_{i}\right)}{\partial \Theta_{i}}
$$

and

$$
\mathcal{B}=\left\{\left(\tau_{d_{i}}, \theta\right) \in\left(C_{m \times n} \times D_{m \times n}\right): \tau_{d_{i}}=\theta\right\},
$$

where $C_{m \times n} \subset R^{m+1 \times n+1} \times R^{m}, D_{m \times n} \subset R^{m+1 \times n+1} \times$ $R^{n}$.

\section{Design of Control System}

Definition:(Passivity) The unlimited workspace of the WMR, the coordination of master/slave robot position, velocity is related to linear combination of angular velocity and designed angle $\Theta_{i}, i=m, s$, then

$$
\begin{aligned}
& \Theta_{m}(t)=\dot{q}_{m}+\Gamma q_{s}, \Theta_{s}(t)=\dot{q}_{s}+\Gamma q_{m} \\
& \dot{\Theta}_{m}(t)=\ddot{q}_{m}+\Gamma \dot{q}_{s}, \dot{\Theta}_{s}(t)=\ddot{q}_{s}+\Gamma \dot{q}_{m},
\end{aligned}
$$

where $\Gamma=\operatorname{diag}\left(\Gamma_{1}, \Gamma_{2}, \cdots, \Gamma_{n}\right) \in R^{m \times n}$ is control gain positive definite matrix.

If system exists with above inputs and satisfies the following condition such that (1)-(2) is said to be passive:

$$
\int_{0}^{t} \Theta_{i}^{T}(t) \widehat{\tau}_{i}(t) d t \geq-\gamma, i=m, s,
$$

where $\tau_{m}=\hat{\tau}_{m}-\tilde{\tau}_{m}, \tau_{s}=\hat{\tau}_{s}-\tilde{\tau}_{s}$ and $\gamma$ is some generic constant.

Assumption 1: The master robot controller $\tau_{m}$, slave robot controller $\tau_{s}$ and $M_{m} \in R^{m \times m}, M_{s} \in R^{n \times n}$ are exists in fixed environment and constant wheel skidsteering is happen, if there exists a constant $C_{\gamma}>0$ such that

$$
\left|2 M_{m} M_{s} \tau_{m} \tau_{s}\right| \leq C_{\gamma}
$$

Assumption 2: If human operator with disturbance of master robot linear velocity and slave robot desired angle with fixed environment effect is happen and error angle $\theta=0$, then

$$
\int_{0}^{t} \tau_{h}(t) \Theta_{d}(t) d t \geq 0
$$

and

$$
-\int_{0}^{t} \tau_{e}(t) \Theta_{c}(t) d t \geq 0
$$

where $\Theta_{d} \in R^{m \times 1}, \Theta_{c} \in R^{n \times 1}$.

\subsection{Feedback passivation}

The master and slave inputs can be defined as

$$
\begin{aligned}
\bar{\tau}_{m}(t) & =-M_{m}\left(q_{m}\right) \Gamma \ddot{q}_{m}-C_{m} \Gamma\left(q_{m}, \dot{q}_{m}\right) q_{m} \\
& +\hat{\tau}_{m}-\tau_{d_{m}}+\tau_{h} \\
\bar{\tau}_{s}(t) & =-M_{s}\left(q_{s}\right) \Gamma \ddot{q}_{s}-C_{s} \Gamma\left(q_{s}, \dot{q}_{s}\right) q_{s} \\
& +\hat{\tau}_{s}-\tau_{d_{s}}-\tau_{e}
\end{aligned}
$$

where $\Gamma=\operatorname{diag}\left(\Gamma_{1}, \Gamma_{2}, \cdots, \Gamma_{n}\right) \in R^{m \times n}$ is control gain positive definite matrix and $\bar{\tau}_{m}, \bar{\tau}_{s}$ are master/slave new state input vectors.

More specifically, there exists a locally Lipschitz function $V: R^{l_{j}} \times R^{m+1 \times n+1} \times R^{m \times n} \times R^{j} \rightarrow R$ such that the following lemma holds.

Lemma 1: Suppose $\theta$ is close to desired input controller $\tau_{d m}, \tau_{d s}$, then equation (4)-(5) are satisfied, if there exists a constant $\gamma_{1}$ such that system (1)-(2) is passive with respect of controller (6) as the following condition hold:

$$
\int_{0}^{t} \theta^{T} \bar{\tau}_{i}(t) d t \geq-\gamma_{1}, i=m, s .
$$

Proof: Consider the kinetic energy of Lyapunov func- 
tion

$$
V(t)=\frac{1}{2} \Theta_{i}^{2}(t)+\frac{1}{2} \theta^{T} B\left(q_{i}\right) \theta, i=m, s,
$$

where $B\left(q_{i}\right)$ is $R^{o \times o}$ matrix and $\dot{\theta}=\dot{\Theta}_{i}-\tau_{i}, \quad i=$ $m, s$.

Differentiating (10) w.r.t, t, we get

$$
\begin{aligned}
\dot{V}(t) & =\Theta_{i} \dot{\Theta}_{i}(t)+\theta^{T} B\left(q_{i}\right) \dot{\theta} \\
& =\Theta_{i}\left[\ddot{q}_{i}(t)+\Gamma \dot{q}_{i}(t)\right]+\theta^{T} B\left(q_{i}\right)\left[\dot{\Theta}_{i}-\tau_{i}\right] \\
& =\Theta_{i}\left[\ddot{q}_{i}(t)+\Gamma \dot{q}_{i}(t)\right]+\theta^{T} B\left(q_{i}\right)\left[\ddot{q}_{m}(t)\right. \\
& +\Gamma \dot{q}_{s}(t)+\ddot{q}_{s}(t)+\Gamma \dot{q}_{m}(t)-\left[-M_{m} \Gamma \dot{q}_{m}(t)\right. \\
& \left.-C_{m} \Gamma q_{m}(t)+\bar{\tau}_{m}\right]-M_{s} \Gamma \dot{q}_{s}(t)-C_{m} \Gamma q_{m}(t) \\
& +\bar{\tau}_{m}-M_{s} \Gamma \dot{q}_{s}(t)-C_{s} \Gamma q_{s}(t)+\bar{\tau}_{s} \\
& =\left[\dot{q}_{m}(t)+\Gamma q_{s}(t)+\dot{q}(t)+\Gamma q_{m}(t)\right] \\
& \times\left[\ddot{q}_{m}(t)+\Gamma \dot{q}_{s}(t)+\ddot{q}_{s}(t)+\Gamma \dot{q}_{m}(t)\right] \\
& -B\left(q_{i}\right)\left[\dot{q}_{m}(t)+\Gamma q_{s}(t)+\dot{q}_{s}(t)+\Gamma q_{m}(t)\right] \\
& \times\left[\ddot{q}_{m}(t)+\Gamma \dot{q}_{s}(t)+\ddot{q}_{s}(t)+\Gamma \dot{q}_{m}(t)\right. \\
& +M_{m} \Gamma \dot{q}_{m}(t)+C_{m} \Gamma q_{m}(t)+\bar{\tau}_{m}+M_{s} \Gamma \dot{q}_{s}(t) \\
& \left.+C_{s} \Gamma q_{s}(t)+\bar{\tau}_{s}\right] \\
& =-B\left(q_{i}\right)\left[\ddot{q}_{m} \bar{\tau}_{m}+\ddot{q}_{m} \bar{\tau}_{s}+2 q_{m} C_{m} \Gamma^{2} q_{s}\right. \\
& \left.+2 q_{s} \Gamma \bar{\tau}_{m}+q_{s}^{2} \Gamma^{2} C_{s}+2 q_{s} \Gamma \bar{\tau}_{s}+\ddot{q}_{s} \bar{\tau}_{m}+\ddot{q}_{s} \bar{\tau}_{s}\right]
\end{aligned}
$$

By using the inequality $2 a b \leq a^{2} \epsilon+\frac{b^{2}}{\epsilon}$, we get

$$
\begin{aligned}
\dot{V}(t) & \geq-B(q)\left[M_{m} \ddot{q}_{m}^{2}+\frac{1}{2 M_{m}} \bar{\tau}_{m}^{2}+M_{m} \ddot{q}_{m}^{2}\right. \\
& +\frac{1}{2 M_{m}} \bar{\tau}_{s}^{2}+C_{m} \Gamma q_{m}^{2}+C_{m} \Gamma q_{s}^{2}+C_{m} \Gamma q_{s}^{2} \\
& +\frac{\Gamma}{C_{m}} \bar{\tau}_{m}+C_{s} \Gamma q_{s}^{2}+C_{s} \Gamma q_{s}^{2}+\frac{1}{C_{s} \Gamma} \bar{\tau}_{s}^{2} \\
& \left.+M_{s} \ddot{q}_{s}^{2}+\frac{1}{2 M_{s}} \bar{\tau}_{m}^{2}+M_{s} \ddot{q}_{s}^{2}+\frac{1}{2 M_{s}} \bar{\tau}_{m}^{2}\right] \\
& \geq-B\left(q_{i}\right)\left[\frac{1}{M_{m}} \tau_{m}+\frac{1}{M_{s}} \tau_{s}\right] \\
& \geq-B\left(q_{i}\right)\left[2 M_{m} M_{s} \tau_{m} \tau_{s}\right] .
\end{aligned}
$$

As per Assumption $1 B\left(q_{i}\right) C_{\gamma} \leq \gamma$, the above lemma is passive.

\subsection{Wheeled mobile robot error dynamical model}

If $\theta$ close to future predicted input $\tau_{d_{i}}, i=m, s$ then $\tau_{d_{i}}=\theta-\tau_{i}, i=m, s$. Let WMR is moving on smooth plane, then $\tau_{d_{i}}$ is constant (i.e. no skidsteering on wheel) and $\theta=\Theta+c$. However,if skidsteering is happen, the future prediction $\tau_{d_{i}}$ is not constant but derivation of $\tau_{d_{i}}$ is bounded,i.e. $\left|\dot{\tau}_{d_{i}}\right| \leq$ $k_{\alpha}, k_{\alpha}>0$. If future input is depending wheel slippage as well as communication error $e_{i}, i=m, s$. For this we assume error $e_{i}, i=m, s$ is difference between future prediction position and as well as current position with respect of skid-steering effect frame respectively.

In above situation, the error $e_{i}, i=m, s$ can be established corresponding to (4) and $\left|\dot{\tau}_{d_{i}}\right|$ is unbounded, then $e_{m}$ and $e_{s}$ can be accumulated as

$$
\begin{aligned}
e_{m} & =\Theta_{m}-\int_{0}^{t} \tau_{d_{m}(t)} d t \\
e_{s} & =\dot{\Theta}_{s}-\int_{0}^{t} \tau_{d_{s}(t)} d t
\end{aligned}
$$

Fig. 2 shows two different framers connecting in Teleoperation task, having clear environment information rather than local information and more effective commands within unlimited workspace of slave robot mapping and limited workspace of human operator to assess the WMRs position, i.e. master robot

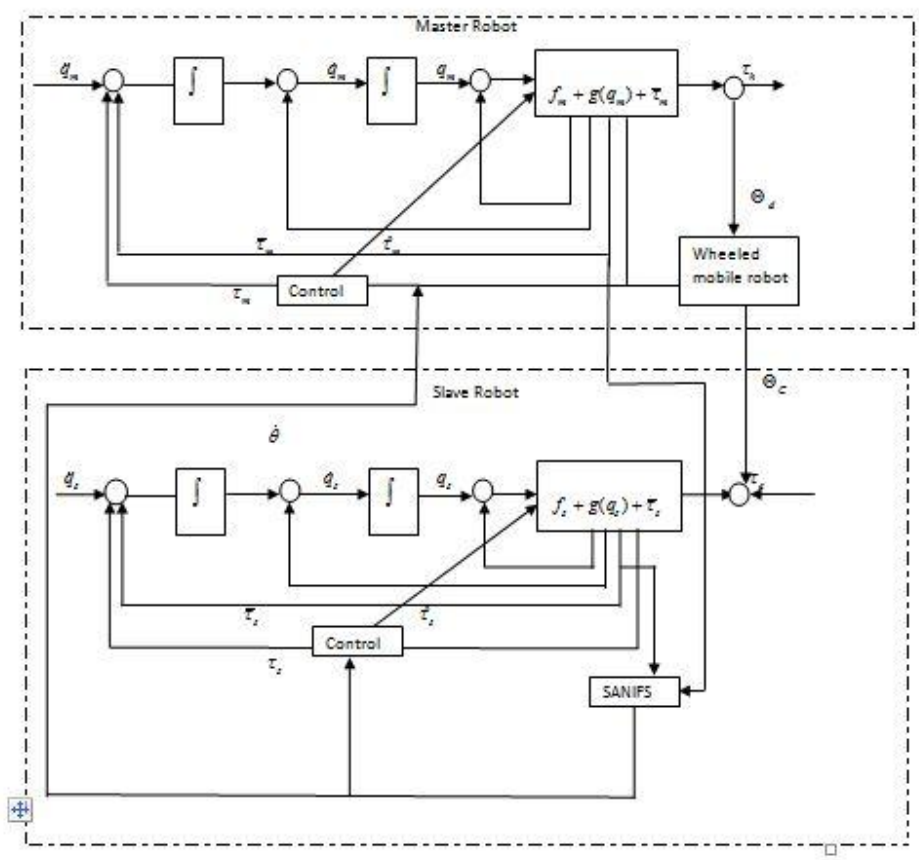

Fig. 2. Control structure of dynamic stabilizer.

Remark 2 In this case $\ddot{\Theta}$ and higher derivatives 
are zero because our system is second order differential system, if you go behind the limit this methodology not support.

\section{Stability Analysis}

In this section stability analysis of system (1)-(2) with implementing hybrid passivity error feedback controller described as follows:

\subsection{Synchronization of master/slave robot error dynamical system}

The important issue that needs is smooth tracking without wheel slippage; hence two dynamical system errors must be synchronized. In this situation as based on equation (7) the coordination $q_{i}, i=m, s$ refluxed to error vector $e_{i}$, then system (9) can be written as

$$
\begin{aligned}
& M_{m}\left(e_{m}\right) \ddot{e}_{m}(t)+C_{m}\left(e_{m}, \dot{e}_{m}\right) e_{m}+g\left(q_{m}\right)+\tau_{d_{m}} \\
& =\tau_{h}+\hat{\tau}_{m} \\
& M_{s}\left(e_{s}\right) \ddot{e}_{m}(t)+C_{s}\left(e_{s}, \dot{e}_{s}\right) e_{s}+g\left(q_{s}\right)+\tau_{d_{s}} \\
& =-\tau_{e}+\hat{\tau}_{s}
\end{aligned}
$$

where $\tau_{d_{m}}, \tau_{d_{s}}, \tau_{h}, \tau_{e}$ are same as equation (1)-(2) and $\hat{\tau}_{m}, \hat{\tau}_{s}$ are defined later.

\subsection{Passivity control design of master/slave robot error dynamical system}

The important issue that needs is smooth tracking without wheel slippage; hence two dynamical system errors must be synchronized. For this we assume $e_{i}, i=m, s$ can be utilized in hybrid system dynamics. In this case we consider error input $e_{i}$ can be written in the suggestive form

$$
\begin{aligned}
& \dot{\tau}_{d_{i}}+e_{i} \in f_{1}\left(\dot{\theta}, \dot{\Theta}_{i}, \tau_{i}\right) \\
& e_{i}=f_{1}\left(\dot{\theta}, \dot{\Theta}_{i}, \tau_{i}\right)-\dot{\tau}_{d_{i}} \\
& \left(\dot{\tau}_{d_{i}}, e_{i}, \dot{\theta}, \dot{\Theta}_{i}, \tau_{i}\right) \in R^{l_{j}} \times R^{o} \\
& \times R^{m+1 \times n+1} \times R^{m \times n} \times R^{j}, j=m, s
\end{aligned}
$$

with output

$$
e_{i}-\dot{\theta}=\tau_{i}-\dot{\tau}_{d_{i}}
$$

$f_{1}: C_{m \times n} \times D_{m \times n} \rightarrow R$ is semi continuous and locally bounded on the compact intervals of $\mathcal{D}$, the inputs $\tau_{m}, \tau_{s}, e_{i}$ and $\tau_{d_{i}}, i=m, s$ exists in $f_{1}$ and satisfies (3) then

$\mathcal{D}=\left\{\left(\dot{\tau}_{d_{i}}+e_{i}, \dot{\theta}\right) \in\left(C_{m \times n} \times D_{m \times n}\right): \dot{\tau}_{d_{i}}+e_{i}=\dot{\theta}\right\}$, where $C_{m \times n} \subset R^{m+1 \times n+1} \times R^{m}, D_{m \times n} \subset R^{m+1 \times n+1} \times$ $R^{n}$.

If $e_{i}, i=m, s$ is utilized in dynamical system, then regulated output controller

$$
\begin{aligned}
\hat{\tau}_{m} & =-M_{m}\left(e_{m}\right) \ddot{e}_{m}-C_{m}\left(e_{m}, \dot{e}_{m}\right) e_{m}-g\left(q_{m}\right) \\
& -\tau_{h}-\int_{0}^{t} \tau_{d_{m}} d t \\
\hat{\tau}_{s} & =-M_{m}\left(e_{s}\right) \ddot{e}_{s}-C_{s}\left(e_{s}, \dot{e}_{s}\right) e_{s}-g\left(q_{s}\right) \\
& +\tau_{e}-\int_{0}^{t} \tau_{d_{s}} d t .
\end{aligned}
$$

Using the Lemma 1, we can analyze the passivity of the proposed controllers is per the following theorem.

Theorem 1: Suppose $\dot{\theta}$ is close to desired input controller(11), equation (4)-(7) are satisfied, under Assumption 1 and 2, the proposed tracking wheeled mobile robot system (1)-(2) is stable in terms of $q_{s} \rightarrow e_{s}, q_{m} \rightarrow e_{m}$ as $t \rightarrow \infty$.

Proof: Consider the semi-definite function

$$
\begin{aligned}
V_{s} & ={ }_{2} e_{m}^{T} k_{m} e_{m}+{ }_{2} e_{s}^{T} k_{s} e_{s} \\
& +\int_{0}^{t} e_{m}^{T} k_{m} e_{m} d s+\int_{0}^{t} e_{s}^{T} k_{s} e_{s} d s .
\end{aligned}
$$

Differentiating (14) w.r.t, t

$$
\begin{aligned}
\dot{V}_{s} & =e_{m}^{T} k_{m}\left(\dot{e}_{m}+e_{s}^{T} k_{s} \dot{e}_{s}+e_{m}^{T} k_{m} e_{m}+e_{s}^{T} k_{s} e_{s}\right. \\
& =e_{m}^{T} k_{m}\left(\dot{e}_{m}+e_{m}\right)+e_{s}^{T} k_{s}\left(\dot{e}_{s}+e_{s}\right) \\
& =e_{m}^{T} k_{m}\left(\tau_{h}+e_{m}-c_{m} e_{m}-\tau_{d_{m}}+\dot{\Theta}_{m}\right. \\
& \left.-\int_{0}^{t} \tau_{d_{m}} d s\right)+e_{s}^{T} k_{s}\left(-\tau_{e}+e_{s}-c_{s} e_{s}\right. \\
& \left.-\tau_{d_{s}}+\dot{\Theta}_{s}-\int_{0}^{t} \tau_{d_{s}} d s\right) \\
& =e_{m}^{T} k_{m}\left[\tau_{h}+e_{m}-c_{m} e_{m}-\tau_{d_{m}}+\ddot{q}_{m}(t)\right. \\
& \left.+\Gamma \dot{q}_{s}(t)-\int_{0}^{t} \tau_{d_{m}} d s\right]+e_{s}^{T} k_{s}\left[-\tau_{e}+e_{s}\right.
\end{aligned}
$$$$
\left.-c_{s} e_{s}-\tau_{d_{s}}+\ddot{q}_{s}(t)+\Gamma \dot{q}_{m}(t)-\int_{0}^{t} \tau_{d_{s}} d s\right]
$$ 


$$
\begin{aligned}
\dot{V}_{s} & =e_{m}^{T} k_{m}\left\{\tau_{h}+\bar{e}_{m}-c_{m} e_{m}-\tau_{d_{m}}\right. \\
& +\frac{1}{M_{m}}\left(-c_{m} q_{m}-\tau_{d_{m}}+\tau_{m}+\tau_{h}\right)+\Gamma \dot{q}_{s} \\
& \left.-\int_{0}^{t} \tau_{d_{m}} d s\right\}+e_{s}^{T} k_{s}\left\{-\tau_{e}+\bar{e}_{s}-c_{s} e_{s}\right. \\
& -\tau_{d_{s}}+\frac{1}{M_{s}}\left(-c_{s} q_{s}-\tau_{d_{s}}-\tau_{e}+\tau_{s}\right) \\
& \left.+\Gamma \dot{q}_{m}-\int_{0}^{t} \tau_{d_{s}} d s\right\} \\
& =e_{m}^{T} k_{m}\left\{\tau_{h}+\bar{e}_{m}-c_{m}\left(q_{m}-q_{m_{d}}+\tau_{m}\right.\right. \\
& \left.-\tau_{m_{d}}\right)-\tau_{d_{m}}+\frac{1}{M_{m}}\left(-c_{m} q_{m}-\tau_{d_{m}}\right. \\
& \left.\left.+\tau_{m}+\tau_{h}\right)+\Gamma \dot{q}_{s}-\int_{0}^{t} \tau_{d_{m}} d s\right\} \\
& +e_{s}^{T} k_{s}\left\{-\tau_{e}+\bar{e}_{s}-c_{s}\left(q_{s}-q_{s_{d}}+\tau_{s}-\tau_{s_{d}}\right)\right. \\
& -\tau_{d_{s}}+\frac{1}{M_{s}}\left(-c_{s} q_{s}-\tau_{d_{s}}-\tau_{e}+\tau_{s}\right) \\
& \left.+\Gamma \dot{q}_{m}-\int_{0}^{t} \tau_{d_{s}} d s\right\} .
\end{aligned}
$$

If $M_{m} \geq 1, M_{s} \geq 1, c_{m} \geq 1, c_{s}, \Gamma \geq 1$, then

$$
\begin{aligned}
\dot{V}_{s} & \leq e_{m}^{T} k_{m}\left\{\bar{e}_{m}-\tau_{d_{m}}+\Gamma \dot{q}_{s}-\int_{0}^{t} \tau_{d_{m}} d s\right\} \\
& +e_{s}^{T} k_{s}\left\{\bar{e}_{s}-\tau_{d_{s}}+\Gamma \dot{q}_{m}-\int_{0}^{t} \tau_{d_{s}} d s\right\} \\
& \leq e_{m}^{T} k_{m}\left\{\bar{e}_{m}+\frac{1}{\Gamma} e_{m}-q_{s}-\tau_{d_{m}}\right\} \\
& +e_{s}^{T} k_{s}\left\{\bar{e}_{s}+\frac{1}{\Gamma} e_{s}-q_{m}-\tau_{d_{s}}\right\} \\
& \leq-e_{m}^{T} k_{m} q_{s}-e_{m}^{T} k_{m} \tau_{d_{m}}-e_{s}^{T} k_{s} q_{m} \\
& -e_{s}^{T} k_{s} \tau_{d_{s}} .
\end{aligned}
$$

Integration (15) on $(0, t)$, we get

$$
\begin{aligned}
V_{s}(t)-V_{s}(0) & \leq-\int_{0}^{t} e_{m}^{T} k_{m} q_{s} d s-\int_{0}^{t} e_{m}^{T} k_{m} \tau_{d_{m}} d s \\
& -\int_{0}^{t} e_{s}^{T} k_{s} q_{m} d s-\int_{0}^{t} e_{s}^{T} k_{s} \tau_{d_{s}} d s(14)
\end{aligned}
$$

We know that $\int_{0}^{t} \Theta_{i}^{T} \widehat{\tau}_{i}(t) d t \geq-\gamma_{1}$ and apply this condition in (16), we get

$$
\begin{aligned}
V_{s}(t)-V_{s}(0) \leq & 2 \gamma_{1}-\int_{0}^{t} e_{m}^{T} k_{m} q_{s} d s \\
& -\int_{0}^{t} e_{s}^{T} k_{s} q_{m} d s .
\end{aligned}
$$

Using Grownwell's inequality for (17), we get

$$
V_{s}(t) \leq e^{-\left(e_{m}^{T} k_{m} q_{s}+e_{s}^{T} k_{s} q_{m}\right)}
$$

If $q_{s} \rightarrow e_{m}, q_{m} \rightarrow e_{s}$ as $t \rightarrow \infty$, therefore the system (1)-(2) is stable.

\subsection{Passivity of adaptive network fuzzy in ference controller}

The robot is moving towards unknown target location with obstacles path. The objective of this control structure is to avoid collision and how to get acquire information form sensor within obstacles environment. Therefore, the necessary control

$$
\begin{aligned}
\tau_{m}= & f_{m}\left(q_{m}, \dot{q}_{m}, \ddot{q}_{m}, \Theta_{m}, \dot{\Theta}_{m}, e_{s}\right) \\
& +g\left(q_{m}\right)+\tau_{d_{m}}+\bar{\tau}_{m} \\
\tau_{s}= & f_{s}\left(q_{s}, \dot{q}_{s}, \ddot{q}_{s}, \Theta_{s}, \dot{\Theta}_{s}, e_{m}\right) \\
& +g\left(q_{s}\right)+\tau_{d_{s}}+\bar{\tau}_{s}
\end{aligned}
$$

guarantees perfect sensor detection of robot system (1)-(2). This controller is directly related to distance between the mobile robot and obstacles with worst environment. It is running based on obstacle information and prevents of mobile robot collisions. The master device causes changing in its velocity and changing the position to suit desired velocity occur $e_{i}, i=m, s$ that is error between desired velocity and actual position. The slave robot is interacting the environment force with force feedback and suit is desert distance between sensor visual to area around the obstacle error $e_{s}$.

\subsection{Simpsons 1/3 rule based adaptive network fuzzy inference system}

In this section adaptive network fuzzy inference system (ANFIS) with weighted average method is briefly explained [5][6][11]. This proposed Simpsons $1 / 3$ rule based adaptive network fuzzy inference system (SANFIS) control is interlinking the following fuzzy rules

Rules: IF $e_{i}, i=m, s$ is $\tau_{m}$ THEN $X_{n}=M_{m} q_{m}+$ $N_{m} \dot{q}_{m}+O_{m} \ddot{q}_{m}+P_{m} \Theta_{m}+Q_{m} \dot{\Theta}_{m}+W_{m} e_{s}$, where $M_{i}$ is $q_{i}, N_{i}$ is $\dot{q}_{i}, O_{i}$ is $\ddot{q}_{i}, P_{i}$ is $\Theta_{i}, Q_{i}$ is $\dot{\Theta}_{i}$ and $W_{i}$ is $e_{i}, i=m, s$. 
IF $e_{i}, i=m, s$ is $\tau_{s}$ THEN $Y_{n}=M_{s} q_{s}+N_{s} \dot{q}_{s}+\quad$-future point error at slave robot $\left.\left.)^{2}\right)\right]$ $O_{s} \ddot{q}_{s}+P_{s} \Theta_{s}+Q_{s} \dot{\Theta}_{s}+W_{s} e_{m}$, where $M_{i}$ is $q_{i}, N_{i}$ is $\quad+g\left(q_{s}\right)$

$\dot{q}_{i}, O_{i}$ is $\ddot{q}_{i}, P_{i}$ is $\Theta_{i}, Q_{i}$ is $\dot{\Theta}_{i}$ and $W_{i}$ is $e_{i}, i=m, s$.

$$
\begin{aligned}
\tau_{m} & =q_{m} \cdot M_{m^{*}}+\dot{q}_{m} \cdot N_{m}+\ddot{q_{m}} \cdot O_{m} \\
& +\Theta_{m} \cdot P_{m}+\dot{\Theta}_{m} \cdot Q_{m}=W_{m} \cdot e_{s} \\
& +g\left(q_{m}\right)+\tau_{d_{m}}+\bar{\tau}_{m} \\
\tau_{s} & =q_{s} \cdot M_{s^{*}}+\dot{q}_{s} \cdot N_{s}+\ddot{q}_{s} \cdot O_{s} \\
& +\Theta_{s} \cdot P_{s}+\dot{\Theta}_{s} \cdot Q_{s}=W_{s} \cdot e_{m} \\
& +g\left(q_{s}\right)+\tau_{d_{s}}+\bar{\tau}_{s} .
\end{aligned}
$$

Based on (19)-(20), if output membership functions are related to errors $e_{i}, i=m, s$, then SANFIS has five layers which are performed as follows;

Layer 1 : In this layer the outputs are depending on control action, where the controls are followed Simpson's 1/3, Simpson's 3/8 and trapezoidal rule. In this paper we followed Simpson 1/3 rule, that is $\tau_{m}=\frac{h}{3}[$ (sensor detected angle at right wheel

- desired position at right wheel $)^{2}$

+3 ((sensor detected angle at left wheel

- desired position at left wheel $)^{2}$

$+($ commented angle right

-robot right wheel angle $)^{2}$

+ (commented angle left

-robot left wheel angle $)^{2}$

+ future point error at master robot

-current position left/right wheel $\left.)^{2}\right]$

$+g\left(q_{m}\right)$

$\tau_{s}=\frac{h}{3}[$ (sensor detected error angle at right wheel

- sensor detected error angle at left wheel $)^{2}$

+3 ((commented right wheel angle

-right wheel velocity/left wheel acceleration $)^{2}$

+ (commented left wheel angle

-left wheel velocity/ right wheel acceleration $)^{2}$

+ (sensor detected error angle at right/left
Layer 2: In this layer the controller is received the outputs as multiplication of inputs to each other. Therefore

$$
\begin{aligned}
\tau_{m} & =q_{m} \cdot M_{m}+\dot{q}_{m} \cdot N_{m}+\ddot{q_{m}} \cdot O_{m} \\
& +\Theta_{m} \cdot P_{m}+\dot{\Theta}_{m} \cdot Q_{m}+W_{m} \cdot e_{s} \\
& +g\left(q_{m}\right)+\tau_{d_{m}}+\bar{\tau}_{m}=X_{n} \\
\tau_{s} & =q_{s} \cdot M_{s^{*}}+\dot{q}_{s} \cdot N_{s}+\ddot{q_{s}} \cdot O_{s} \\
& +\Theta_{s} \cdot P_{s}+\dot{\Theta}_{s} \cdot Q_{s}+W_{s} \cdot e_{m} \\
& +g\left(q_{s}\right)+\tau_{d_{s}}+\bar{\tau}_{s}=Y_{n} .
\end{aligned}
$$

Here $X_{n}$ and $Y_{n}$ are truth value of output mesh point. Layer 3 : This layer is normalization layer, it could be rearranged all outputs in SANFIS. Therefore

$$
\bar{X}_{n}=\frac{X_{n}}{X_{n}-\tau_{d_{m}}}, \quad \bar{Y}_{n}=\frac{Y_{n}}{Y_{n}-\tau_{d_{s}}}
$$

Layer 4: This layer is called adaptive output node whose output is product of normalized firing strength. A defuzzification node determines the weighted consequence of the output and connection between input and output, which is written as

$$
\begin{gathered}
\frac{X_{n}}{X_{n}-\tau_{d_{m}}}+g\left(q_{m}\right)+\tau_{d_{m}}+\bar{\tau}_{m}=\bar{X}_{n}\left(q_{m} \cdot M_{m}\right. \\
+\dot{q}_{m} \cdot N_{m}+\ddot{q}_{m} \cdot O_{m}+\Theta_{m} \cdot P_{m}+\dot{\Theta}_{m} \cdot Q_{m} \\
\quad+W_{m} e_{s}+g\left(q_{m}\right)+\tau_{d_{m}}+\bar{\tau}_{m} \\
\frac{Y_{n}}{Y_{n}-\tau_{d_{s}}}+g\left(q_{s}\right)+\tau_{d_{s}}+\bar{\tau}_{s}=\bar{Y}_{n}\left(q_{s} \cdot M_{s}\right. \\
+\dot{q}_{s} \cdot N_{s}+\ddot{q}_{s} \cdot O_{s}+\Theta_{s} \cdot P_{s}+\dot{\Theta}_{s} \cdot Q_{s} \\
+W_{s} e_{m}+g\left(q_{s}\right)+\tau_{d_{s}}+\bar{\tau}_{s}
\end{gathered}
$$

Layer5: This node is fixed node and sum of determines of output deffuzzification is equivalent to overall system output with steering angles, that is

$$
\begin{gathered}
\sum_{i=m, s} \bar{X}_{n} \cdot \Theta_{i}+g\left(q_{m}\right)+\tau_{d_{m}}+\bar{\tau}_{m}=\frac{\sum_{i=m, s} \bar{X}_{n} \cdot \Theta_{i}}{\sum_{i=s, m} X_{n} \cdot \dot{\Theta}_{i}} \\
\quad+g\left(q_{m}\right)+\tau_{d_{m}}+\bar{\tau}_{m},
\end{gathered}
$$

$$
\sum_{i=m, s} \bar{Y}_{n} \cdot f_{r}+g\left(q_{s}\right)+\tau_{d_{s}}+\bar{\tau}_{s}=\frac{\sum_{i=m, s} \bar{Y}_{n} \cdot \Theta_{i}}{\sum_{i=s, m} Y_{n} \cdot \dot{\Theta}_{i}}
$$




$$
+g\left(q_{s}\right)+\tau_{d_{s}}+\bar{\tau}_{s}
$$

To train the learning process of safe navigation, the actual coordinate sensor data and desired angle data set is used SANFIS while the testing dataset is verifying Simpson'1/3 rule due to accuracy and effectiveness. The specification of the proposed control structure is demonstrated in simulation.

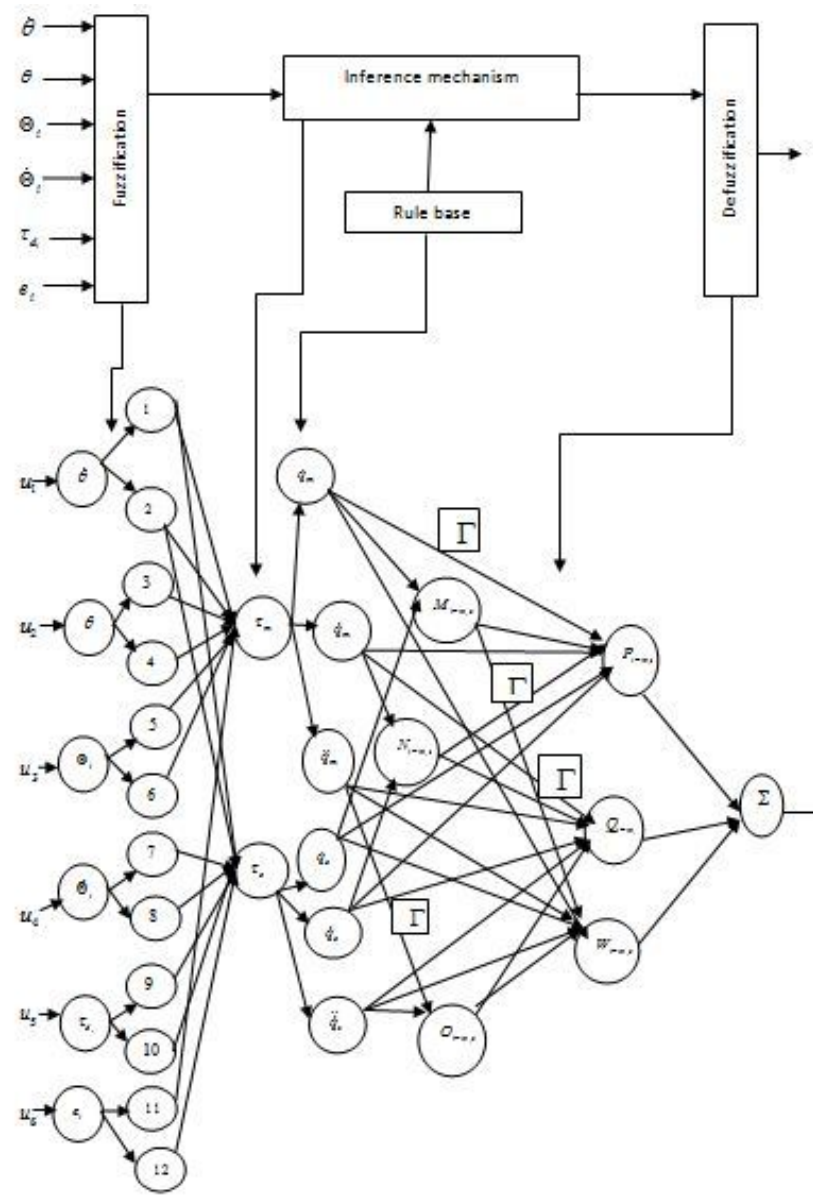

Fig. 3. The proposed SANFIS structure: in this figure 1.sensor detected angle at right wheel, 2.sensor detected angle at right wheel, 3 . commented right wheel angle, 4. commented left wheel angle, 5. current position of right wheel/desired position of right wheel, 6 . current position of left wheel/desired position of left wheel, 7.right wheel velocity/left wheel acceleration, 8.left wheel velocity/right wheel acceleration, 9.future point on right wheel, 10.future point on left wheel, 11.future point master robot error, 12.future point slave robot error

\section{Simulation Result}

In order to achieve the smooth and stable movement of wheeled mobile robot we used teleoperation system with designed controller as proposed earlier. The master robot generate the path for the mobile robot while slave robot to follows without navigation with respect of sensor device response. The sensor data is working on obstacle avoidance with respect of Simpsons 1/3 rule based adaptive network fuzzy inference system(SANFIS). The dynamical model of the mobile robot in global coordination can be reformulated via Simpsons $1 / 3$ rule method as $X_{n}=$ $\frac{\tau_{m}}{\frac{\dot{q} m r-q_{m l}}{m l}}$, where $\dot{q}_{m r}, q_{m l}$ right wheel velocity and left wheel position of master robot and $m_{l}$ is distance between two wheels and $\bar{X}_{n}=\frac{\tau_{m}}{\frac{\bar{q}_{m l}-q_{m r}}{m l}}$, left wheel velocity and right wheel position of master robot respectively. Similarly $Y_{n}=\frac{\tau_{s}}{\frac{\dot{q}_{s r}-q_{s l}}{m l}}$, where $\dot{q}_{s r}, q_{s l}$ right wheel velocity and left wheel position of slave robot and $m_{l}$ is distance between two wheels and $\bar{Y}_{n}=\frac{\tau_{s}}{\frac{\bar{q}_{s l}-q_{s r}}{m l}}$, left wheel velocity and right wheel position of slave robot.

Forward and backward movements will be interpreted to moving position of control device, the negative direction on $Y$-axis / $X$-axis. Consequently, mobile robot right hand coordinated is observed by $\tau_{m}$ with respect of position and wheel slippage, that is moving of right hand is controlled by left hand of $\tau_{s}$. Movement of mobile robot left hand positive to decided point is controlled by $\tau_{s}$ with respect of negative direction on the $X-\operatorname{axis} / Y-$ axis. The simulation results of ANFIS and SANFIS models path segments with master/slave contact mode position and torque tracking profiles are given in Fig.4 and Fig.5, it shows that the mobile robot can follow the path as stable with respect of minimum error.

\section{Comparison of the Two Approaches}

To demonstrate the characteristics of the proposed system, experiments were carried to assess both adaptive network fuzzy inference system(ANFIS) and Simpsons $1 / 3$ rule based adaptive network fuzzy inference system(SANFIS). The adaptive techniques can be used to generate the membership function and fuzzy system delivers the best result from dataset, the results are shown in Fig.4 and Fig.5 and right/left 


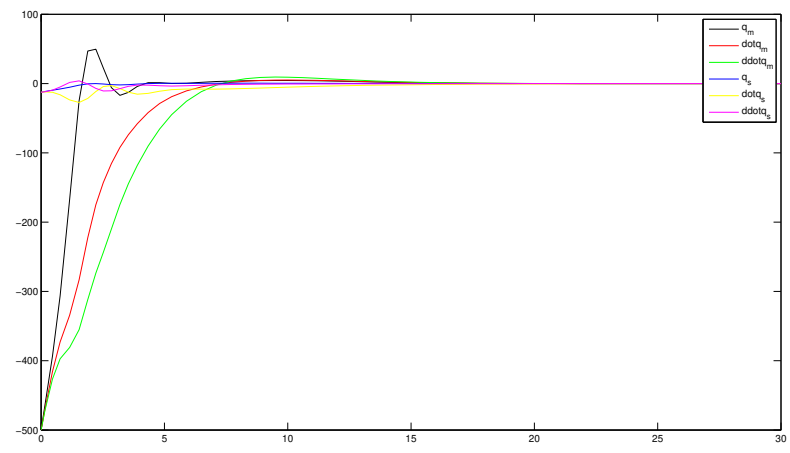

Fig. 4. The error response of right/left coordination of master/slave robot with ANFIS

wheel error angle response is shown in Fig.6 and Fig.7.

The Simpsons $1 / 3$ with adaptive neural network fuzzy inference system (SANFIS) approach requires by far the shortest pre-training time almost within 15 seconds all the trajectories are convergent. In this sense, SANFIS is the best approach and performance measures are tabulated in Tables 1-4. In Table 1 compares another distinguishing property of the approaches namely the need for pre-training, the error bound for Master/Slave robots with left/right wheel angles are given in Table 2. Table 3 and 4 shows the Position, velocity and angular velocity of Master/Slave with right/left wheel response respectively. In this table, the SANFIS approach requires by far the minimum pre-training time. As depicted, the proposed controllers can behave correctly in all cases and this task is realized efficiently.

\section{Conclusions}

Robust, fast and minimal error converge control is proposed for a teleoperation based wheeled mobile robot system under passivity with hybrid system that can achieve perfect tracking and transparency. This theoretical analysis shown the force felt by the human operator is almost equal to the force applied by the predictors environment and sensor data slave robot. The control scheme is provided to perfect position tracking of the master/slave robot with sense of

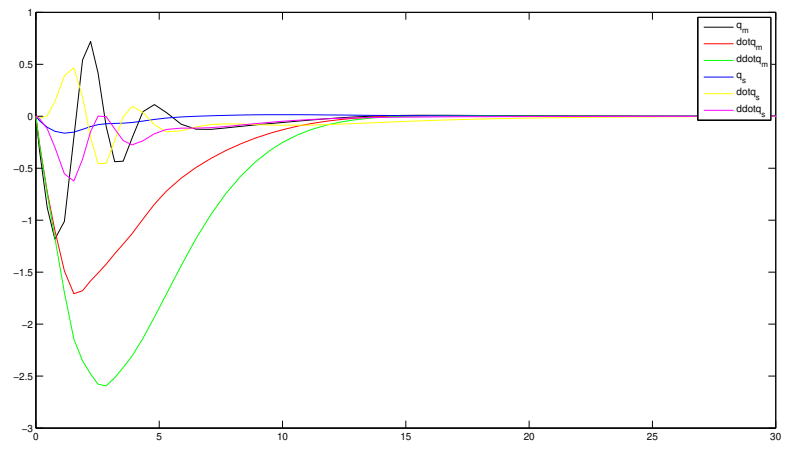

Fig. 5. The error response of right/left coordination of master/slave robot with SANFIS

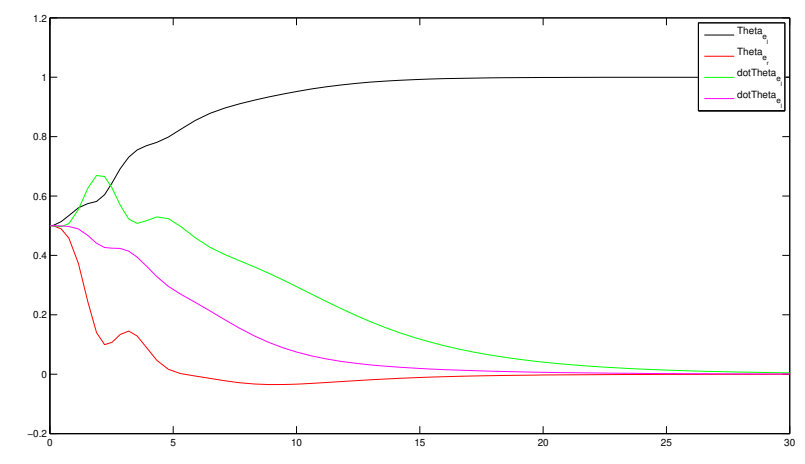

Fig. 6. The error angle response of right/left wheel with ANFIS

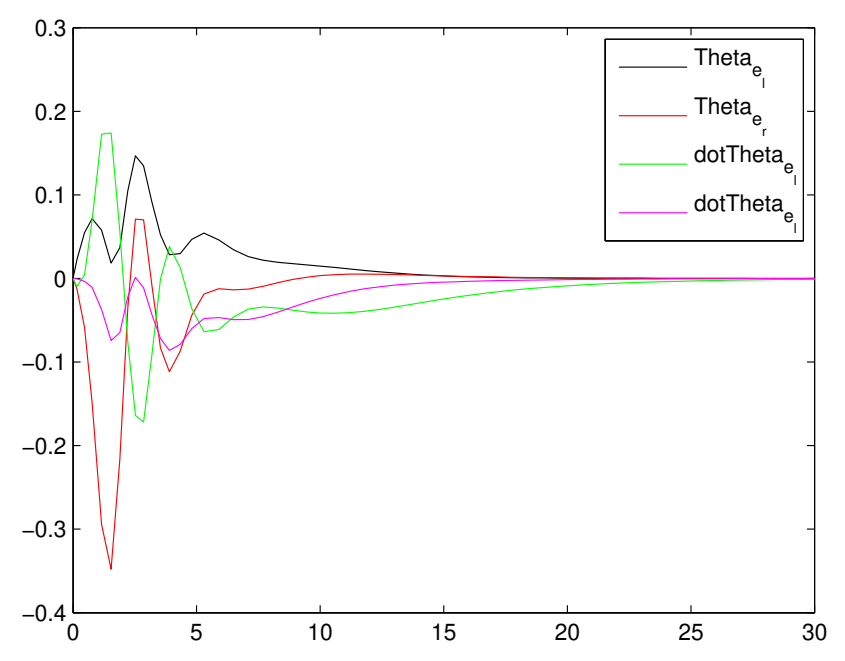

Fig. 7. The error angle response of right/left wheel with SANFIS 
teleoperation in the environment. A soft-computing based adaptive network fuzzy inference system can select the suitable steering angle by Simpson's $1 / 3$ rule to achieve collision-free path while moving to reach the goal. The ANIFS and SANFIS approaches are tested for the same command signal. For the tracking error performance, SANFIS showed the best performance. On the other hand, designed controller provided passivity to the system and stability to the wheeled mobile robot. The simulation result exhibited the efficiency of the proposed controller system. It is believed that future research in this area will also be very promising because of efficient sensor incoming/outgoing signals, demands and robustness in adaptive network fuzzy inference systems, which can benefit from four wheel mobile robot robustness and fast responsiveness of sliding mode control.

\section{REFERENCES}

[1] D.Lee, O.Martinez-palafox, M.W.Spong, "Bilateral teleoperation of a wheeled mobile robot over delayed communication network", Proc. of the IEEE Int. Conf. on Robotics and Automation, 2006, pp.3298-3303, Orlando, FL.

[2] D.Lee, D.Xu, "Feedback $r$-passivity of Lagrangian systems for mobile robot teleoperation", Proc. of the IEEE Int. Conf. on Robotics and Automation, 2006, pp.2118-2123, Shanghai, China.

[3] S.Park, C.Seo, J-P.Kim, J-H. Ryu, "Robust stable rate-mode bilateral teleoperation using an energy bounding approach", Mechatronics, vol.21, 2011, pp.176-184.

[4] I.Farkhatdinov, J-H.Ryu, J.Poduraev, "A feasibility study of time domain passivity approach for bilateral teleoperation of mobile manipulator, Proc. of the IEEE/ICROS, Int. Conf. on Control, Automation and system, 2008, Seoul, Korea.

[5] J.Artigas, J-H.Ryu, C.Preusche and G.Hirzinger, "Network representation and passivity of delayed teleoperation system", Proc. of the IEEE/RSJ Int. Conf. on Intelligent Robots and Systems, 2011, pp.177-183, California.

[6] J.H.Ryu, J.Artigas, C.Preusche, "A passivity bilateral control scheme for a teleoperation with time varying communication delay", Mechatronics, vol.20, 2010, pp.812-823.

[7] Tanboud K. Khaldoun K and S.N.A. Munaf, "A Neural Fuzzy Reasoning System for mobile robot navigation", Jordan Journal of Mechanical Industrial Engg., vol.3. Pp.77-88, 2009.

[8] K.Suganya and V.Arulmozhi, "Soft Computing Controller based Path Planning Wheeled Mobile Robot", IEEE International Conference on Advances in Computer Applications (ICACA), pp.230-234, 2016.

[9] D. Janglova, "Neural Networks in Mobile Robot Motion", International Journal of Advanced Robotic System, vol.1 No 1, pp.15-22, 2004.

[10] J.H.Lilly, "Evolution of a negative-rule fuzzy obstacle avoidance controller for an autonomous vehicle,"IEEE Trans.Fuzzy Syst., vol.15,no.4, pp.718-728, 2007.

[11] J. Shing, R.Jang, "ANFIS: Adaptive-network-based fuzzy inference system", IEEE Trans. on syst., Man and Cybernetics, vol.23, no.3, pp.665-685, 1993.
[12] Xinwu Liang, Hesheng Wang, "Adaptive Image-Based Trajectory Tracking Control of wheeled Mobile Robots with an Uncalibrated Fixed Camera" IEEE Transaction on Control Systems Technology, vol.23, pp.2266-2282, 2015.

[13] Weihua Li, Liang Ding,Zhen Liu, Widong Wang, Haibo Gao and Mahdi Tavakoli ,"Kinematic Bilateral Tele-Driving of Wheeled Mobile Robots Coupled with Slippage", IEEE Transactions on Industrial Electronics, vol.64, pp.2147-2157, 2017.

[14] Weihua Li, Haibo Gao,Liang Ding and Mahdi Tavakoli, "Trilateral Predictor-Mediated Teleoperation of a wheeled Mobile Robot With Slippage", IEEE Robotics and Automation Letters, vol.1, pp.738-745, 2016.

[15] Sung Jin Yoo and Bong Seok Park, "Connectivity-Preserving Approach for Distributed Adaptive Synchronized Tracking of Networked Uncertain Nonholomic Mobile Robots", IEEE Transactions on Cybernetics, vol.48, pp. 2598-2608, 2018

[16] X. Wu,Y. Wang, X. Dang, Robust adaptive sliding-mode control of condenser-cleaning mobile manipulator using fuzzy wavelet neural network, Fuzzy sets and Sys., vol.235, pp.62-82, 2014

[17] M.H.Yamchi and R.M.Esfanjani, "Distributed predictive formation control of networked mobile robots subject to communication delay", Robot. Auton. Syst., vol.75, pp.194-207, 2017.

\begin{tabular}{|c|c|}
\hline Time $(\mathrm{Sec})$ & Model \\
\hline ANFIS & $20-25$ \\
\hline SANFIS & $15-20$ \\
\hline
\end{tabular}

Table.1 : Comparison of pre-training time convergences.

\begin{tabular}{|c|c|c|}
\hline & $\theta$ & $\dot{\theta}$ \\
\hline ANFIS & 0.9999 & 0.0047 \\
\hline SANFIS & $4.8529 \mathrm{e}-06$ & $1.5817 \mathrm{e}-04$ \\
\hline
\end{tabular}

Table.2 : Comparison two methods ANFIS and SANFIS with left/right commented angle and sensor detected angle left/right.

\begin{tabular}{|c|c|c|c|}
\hline & $q_{m}$ & $\dot{q}_{m}$ & $\ddot{q}_{m}$ \\
\hline ANFIS & $-4.7521 \mathrm{e}-04$ & -0.0058 & 0.0064 \\
\hline SANFIS & $-7.5854 \mathrm{e}-05$ & $-2.1220 \mathrm{e}-05$ & $-4.8266 \mathrm{e}-05$ \\
\hline
\end{tabular}

Table. 3 : Comparison of ANFIS and SANFIS with coordination of master robot.

\begin{tabular}{|c|c|c|c|}
\hline & $q_{s}$ & $\dot{q}_{s}$ & $\ddot{q}_{s}$ \\
\hline ANFIS & -0.0016 & -0.0661 & -0.0103 \\
\hline SANFIS & $8.7194 \mathrm{e}-05$ & -0.0020 & $-3.1626 \mathrm{ee}-04$ \\
\hline
\end{tabular}

Table.4 : Comparison of ANFIS and SANFIS with coordination of slave robot.

\section{Creative Commons Attribution License 4.0 (Attribution 4.0 International, CC BY 4.0)}

This article is published under the terms of the Creative Commons Attribution License 4.0

https://creativecommons.org/licenses/by/4.0/deed.en US 